\title{
Substrate effects on the micro/nanomechanical properties of TiN coatings
}

\author{
T.H. Zhang* and Y. Huan \\ State Key Laboratory of Nonlinear Mechanics (LNM), Institute of Mechanics, Chinese Academy of Sciences, \\ Beisihuan West Road 15, Beijing 100080, China
}

Received 10 October 2003; accepted 11 June 2004

\begin{abstract}
Nanoindentation and nanoscratch tests were performed for titanium nitride (TiN) coatings on different tool steel substrates to investigate the indentation/scratch induced deformation behavior of the coatings and the adhesion of the coating-substrate interfaces and their tribological property. In this work, TiN coatings with a thickness of about $500 \mathrm{~nm}$ were grown on GT35, 9Cr18 and 40 CrNiMo steels using vacuum magnetic-filtering arc plasma deposition. In the nanoindentation tests, the hardness and modulus curves for TiN/GT35 reduced the slowest around the film thickness $500 \mathrm{~nm}$ with the increase of indentation depth, followed by TiN/9Cr18 and TiN/40CrNiMo. Improving adhesion properties of coating and substrate can decrease the differences of internal stress field. The scratch tests showed that the scratch response was controlled by plastic deformation in the substrate. The substrate plays an important role in determining the mechanical properties and wear resistance of such coatings. TiN/GT35 exhibited the best load-carrying capacity and scratch/wear resistance. As a consequence, GT35 is the best substrate for TiN coatings of the substrate materials tested.
\end{abstract}

KEY WORDS: nanoindentation, nanoscratch, titanium nitride, mechanical properties, adhesion, wear resistance

\section{Introduction}

Owing to its excellent mechanical properties and wear resistance, titanium nitride (TiN) is commercially used on steels as a protective film to extend their working lifetime. The properties of TiN vary with preparation techniques, deposition conditions and substrates. Therefore, a thorough understanding of the mechanical response of a coated component to applied loads is of both fundamental and technical importance to evaluate the surface hardening and interfacial bonding strength for different materials. The deposition techniques and resulting coating properties have been studied extensively [1-4]. The mechanical properties of the coating are strongly influenced by the substrate on which it are deposited [5,6]. The nanoindentation and nanoscratch techniques are commonly used for evaluating the mechanical properties of such sub-micron coatings.

In the present research, a systematic investigation of the mechanical properties of TiN coatings on different steel substrates was performed. The aim of this study is to understand the predominant deformation mechanism that occurred in response to indentation and scratching. It then critically assesses the ways in which the information can be used to understand the deformation of these coatings.

\footnotetext{
*To whom correspondence should be addressed
}

E-mail: zhangth@1nm.imech.ac.cn

\section{Experimental details}

The substrate materials were GT35 alloy tool steel (with composition in wt.\%: Fe 60, TiC 35, Cr 2, Mo 2, C 1), 9Cr18 bearing steel (Si 0.8, Mn 0.72, P 0.035, $\mathrm{S}$ 0.03, C 0.96, Cr 17.8, Fe 79.655), and 40CrNiMo alloy structure steel (Si 0.25, Mn 0.70, C 0.41, Cr 0.82, Ni 1.45, Mo 0.18, Fe 96.19). The specimens were cut from real bearing rings that had been quenched and tempered. All samples were polished to a mirror finished surface and ultrasonically cleaned prior to coating deposition. TiN coatings with a thickness of about $0.5 \mu \mathrm{m}$ were deposited onto the substrates using the vacuum magnetic-filtering arc plasma method. Finally, a DJB-823 organic film (Institute of Chemical Prevention, Beijing University of Posts and Telecommunication, Beijing, China) of approximately $1.0 \mu \mathrm{m}$ in thickness was deposited on the TiN. The organic film is a solid film protective agent, and plays a lubrication role.

Nanoindentation tests were conducted using a MTS Nano Indenter ${ }^{\circledR}$ XP (MTS Systems Corp., Oak Ridge, TN, USA) with a Berkovich diamond tip. Hardness and elastic modulus were measured using the continuous stiffness measurement (CSM) option. Fused silica was used as a standard sample for the initial calibration. In the present study, a typical indentation experiment consists of seven subsequent steps: approaching the surface; determining the contact point; loading to 
peak load; holding the tip for $10 \mathrm{~s}$ at the peak load; unloading $90 \%$ of peak load; holding the tip for $50 \mathrm{~s}$ at $10 \%$ of the peak load for thermal drift correction; and finally unloading completely. The experimental parameters were chosen as follows: strain rate, $0.05 \mathrm{~s}^{-1}$; allowable thermal drift rate, $0.05 \mathrm{~nm} / \mathrm{s}$; depth limit, $1.0 \mu \mathrm{m}$. The hardness and elastic modulus were obtained from the curves using the Oliver-Pharr method [7]. Nanoscratch tests were performed using the lateral load measurement (LFM) option of the Nano Indenter ${ }^{\circledR}$ XP. A different diamond Berkovich tip was used with face forward. During a scratch test, the ramp normal load on the indenter was held. For these tests, the ramping normal loads were $\max 40$, 100 and $300 \mathrm{mN}$. A scratch velocity of $10 \mu \mathrm{m} / \mathrm{s}$ was used by controlling the stage movement. A typical scratch experiment is performed in nine subsequent steps: approaching the surface; first profile with normal load $20 \mu \mathrm{N}$ for $700 \mu \mathrm{m}$ in $Y$ direction; return profile; pre-profile for $100 \mu \mathrm{m}$; scratch profile with ramping normal load for length $500 \mu \mathrm{m}$; unload; postprofile for $100 \mu \mathrm{m}$; return profile; final profile with normal load $20 \mu \mathrm{N}$ for $700 \mu \mathrm{m}$. Five indents or scratches were performed for each test conditions. All tests were carried out at room temperature. Surface observation was performed by a POLYVAR MET $^{\circledR}$ optical microscope (Reichert-Jung, Vienn Austria).

\section{Results and discussion}

\subsection{Nanoindentation properties}

Nanoindentation tests with max indentation depth $1000 \mathrm{~nm}$ were performed for the steel samples $(40 \mathrm{CrN}$ iMo, 9Cr18, GT35) and the coatings (TiN/40CrNiMo, TiN/9cr18, TiN/GT35). The results are shown in table $1 . p_{\mathrm{m}}, \delta_{\mathrm{m}}, \delta_{\mathrm{r}}, H, E$ are the peak load on sample, max indentation depth, and residual indentation depth, hardness and modulus from unload, respectively. At the same depth $(1000 \mathrm{~nm})$ for all samples, $p_{\mathrm{m}}$ exhibits the load-carrying capacity; $1-\delta_{\mathrm{r}} / \delta_{\mathrm{m}}$, the indentation recovery capacity. $H / E$ can provide important information on the mechanical resistance of the material. The relative high value of $H / E$ ratio reveals the high elastic deformation during contact, making it an excellent candidate for applications where both wear resistance and elasticity are required [8]. It has been found that the max load, $1-\delta_{\mathrm{r}} / \delta_{\mathrm{m}}$, hardness, modulus, and $H / E$ of GT35 were highest, followed by 9Cr18 and 40CrNiMo. In general, the harder the substrate, the better are the load-carrying capacity and wear resistance. Thus, GT35 steel is the best substrate material. In addition, the residual indentation depths for $\mathrm{TiN} /$ steels were shallower than for the related steels. As a consequence, the TiN film exhibits a better elastic recovery capacity.

Figure 1 shows the hardness values as a function of indentation depth for all above samples. Each point represents the average of five separate indents, and the error bars were calculated from the standard deviation of these five results. The error values could be correlated with the non-homogeneous component distribution in the samples. Note the hardness in the topmost $20 \mathrm{~nm}$. This is probably an artifact of the data reduction method--when the indentation depth is sufficiently shallow, the load-depth curve shows almost complete elastic recovery [9]. Variations in surface topography also led to the scatter in measured values within $50 \mathrm{~nm}$ of the film surface [10]. The hardness values of $40 \mathrm{CrN}$ iMo, $9 \mathrm{Cr} 18$ and GT35 samples were approximately independent of indentation depths. Their hardness and modulus values at $1000 \mathrm{~nm}$ depth are shown in table 1 . In contrast, those of TiN/GT35, TiN/9Cr18, and $\mathrm{TiN} / 40 \mathrm{CrNiMo}$ were dependent on indentation depths. In any coatings, all data show max hardness of about $38 \mathrm{GPa}$ at depths from $50 \mathrm{~nm}$ to $100 \mathrm{~nm}$, consistent with the general practice of keeping depth $<20 \%$ of the film thickness to minimize substrate effects [11]. Therefore, the hardness values of TiN films on the three substrates were approximately $38 \mathrm{GPa}$. With the depth increasing, the hardness values decrease due to substrate effects, and gradually approach those of the corresponding steel substrates. Among the three coatings, the hardness curve for TiN/ GT35 reduced the most slowly around the film thickness $500 \mathrm{~nm}$ with increasing indentation depth. It is shown that the mechanical properties of GT35 steel and TiN film corresponding the most closely. That is, the interface properties change only gradually. The deformation around the TiN/GT35 interface is more consistent under the load. The internal stress field around interface doesn't change abruptly, which

Table 1.

The load/unload-depth data of each sample and the corresponding hardness, and modulus and ratio of $H / E$.

\begin{tabular}{lccccccc}
\hline & $\mathrm{P}_{\mathrm{m}} / \mathrm{mN}$ & $\delta_{\mathrm{m}} / \mathrm{nm}$ & $\delta_{\mathrm{r}} / \mathrm{nm}$ & $1-\delta_{\mathrm{r}} / \delta_{\mathrm{m}} \mid \%$ & Hardness/GPa & Modulus/GPa & $H / E$ \\
\hline GT35 & 250 & 1000 & 760 & 19 & 14.3 & 368.5 & 0.039 \\
TiN/GT35 & 300 & 1000 & 620 & 38 & & & \\
9Cr18 & 200 & 1000 & 800 & 17 & 9.1 & 266.8 & 0.034 \\
TiN/9Cr18 & 250 & 1000 & 640 & 36 & 5.8 & 203.3 & 0.029 \\
40CrNiMo & 120 & 1000 & 840 & 15 & & & \\
TiN/40CrNiMo & 160 & 1000 & 800 & 20 & & & \\
\hline
\end{tabular}




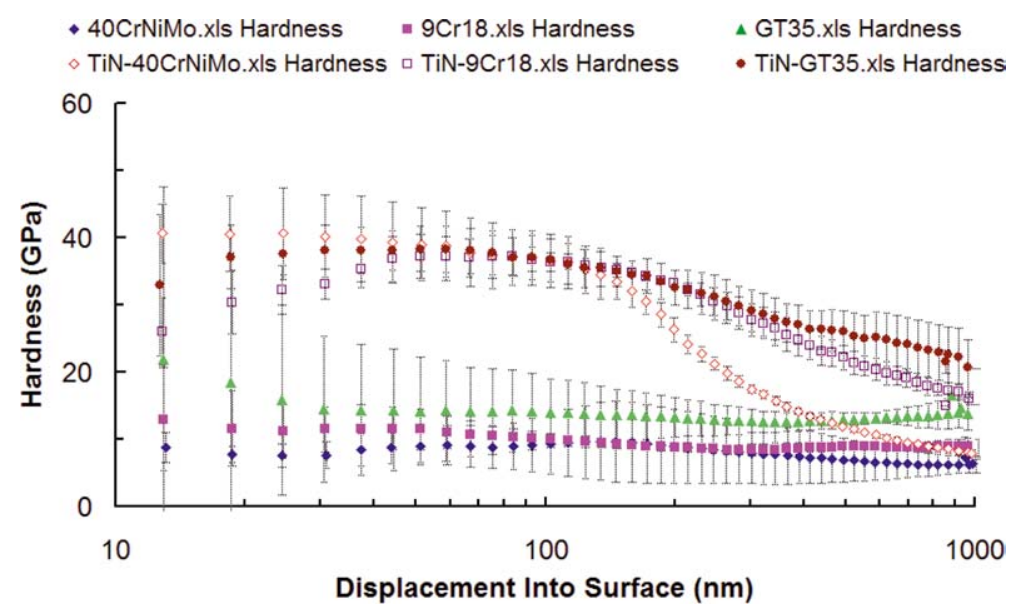

Figure 1. Hardness as a function of displacement into surface curves with five separate indents to about $1000 \mathrm{~nm}$ into the six samples: 40CrNiMo, 9Cr18, GT35, TiN/40CrNiMo, TiN/9Cr18, TiN/GT35 (about 500 nm-thickness TiN).

improves adhesion properties of coating and substrate. So, the combination of coating/substrate properties is important in determining the overall mechanical response of the system.

\subsection{Nanoscratch properties}

To explain the scratch measurement process, the TiN/9Cr18 example is shown in figure 2 for a ramping normal load of $\max 100 \mathrm{mN}$. In figure 2(a), the firstprofile curve corresponds to the roughness of the initial surface, the scratch-profile curve corresponds to the tip penetration depth during testing, and the finalprofile curve represents the residual depth after
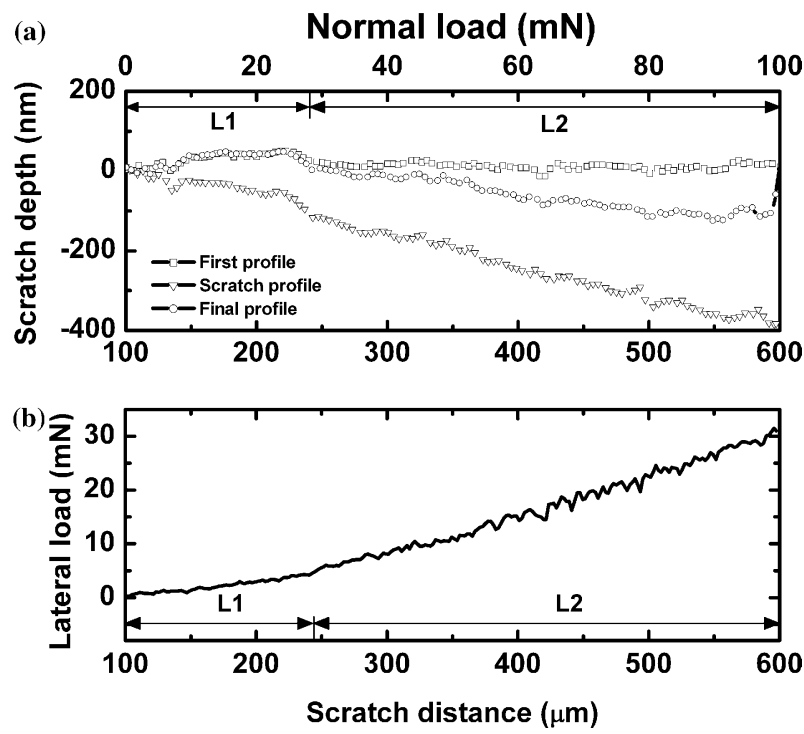

Figure 2. The scratch with a ramping normal load of $\max 100 \mathrm{mN}$ for TiN/9Cr18: (a) The scratch depth as a function of the scratch distance and normal load; (b) The lateral load as a function of the scratch distance and normal load. scratching, assessing the surface damage and any elastic/plastic deformation. A negative depth corresponds to the scratch tip being pushed into the sample. It is evident that the scratch profile increases linearly as a function of the ramping increase of the normal load from 0 to $28 \mathrm{mN}$ or scratch distance from $100 \mu \mathrm{m}$ to $240 \mu \mathrm{m}$, marked as range L1. The final-profile curve nearly coincides with the first-profile over the range L1, although the scratch depth goes up to $100 \mathrm{~nm}$. This indicates that the TiN coatings almost fully recover at these loads, in which the initial elasticity is coating-dependent and is hardly related to the substrate. The coefficient of friction remain constant at 0.15 in figure 3(c). Then over the range $\mathrm{L} 2$ from $240 \mu \mathrm{m}$ to $600 \mu \mathrm{m}$, the slope of the scratch-profile curve increases, and the final-profile curve separates from the first-profile curve. It indicates that the substrate and TiN coating deformed plastically under these loads. The related coefficient of friction increases gradually until approaching the curves of its substrate 9Cr18 (see figure 3(a) and (c)). It is shown that the tip scratched into the substrate. Both lateral load as a function of the normal load and the scratch distance during the scratch profile are shown in figure 2(b).

Figure 3 shows the coefficient of friction as a function of the scratch distance under various ramping normal loads: (a) $9 \mathrm{Cr} 18$ with $40 \mathrm{mN}$; (b) $\mathrm{TiN} / 9 \mathrm{Cr} 18$ with $40 \mathrm{mN}$; (c) TiN/9Cr18 with $100 \mathrm{mN}$; (d) organic/ $\mathrm{TiN} / 9 \mathrm{Cr} 18$ with $100 \mathrm{mN}$; (e) organic/TiN/9Cr18 with $300 \mathrm{mN}$. The friction coefficients of $9 \mathrm{Cr} 18$, TiN film, and organic film are approximately $0.40,0.15$ and 0.11 , respectively. The friction coefficients of GT35, $40 \mathrm{CrNiMo}, \mathrm{TiN}$, and organic film are approximately $0.25,0.45,0.15$, and 0.11 , respectively. Thus, the solid lubricative effects are very marked.

In order to understand the deformation mechanism of the organic/TiN/GT35, organic/TiN/9Cr18, organic/ TiN/40CrNiMo, it needs to be recognized that three 

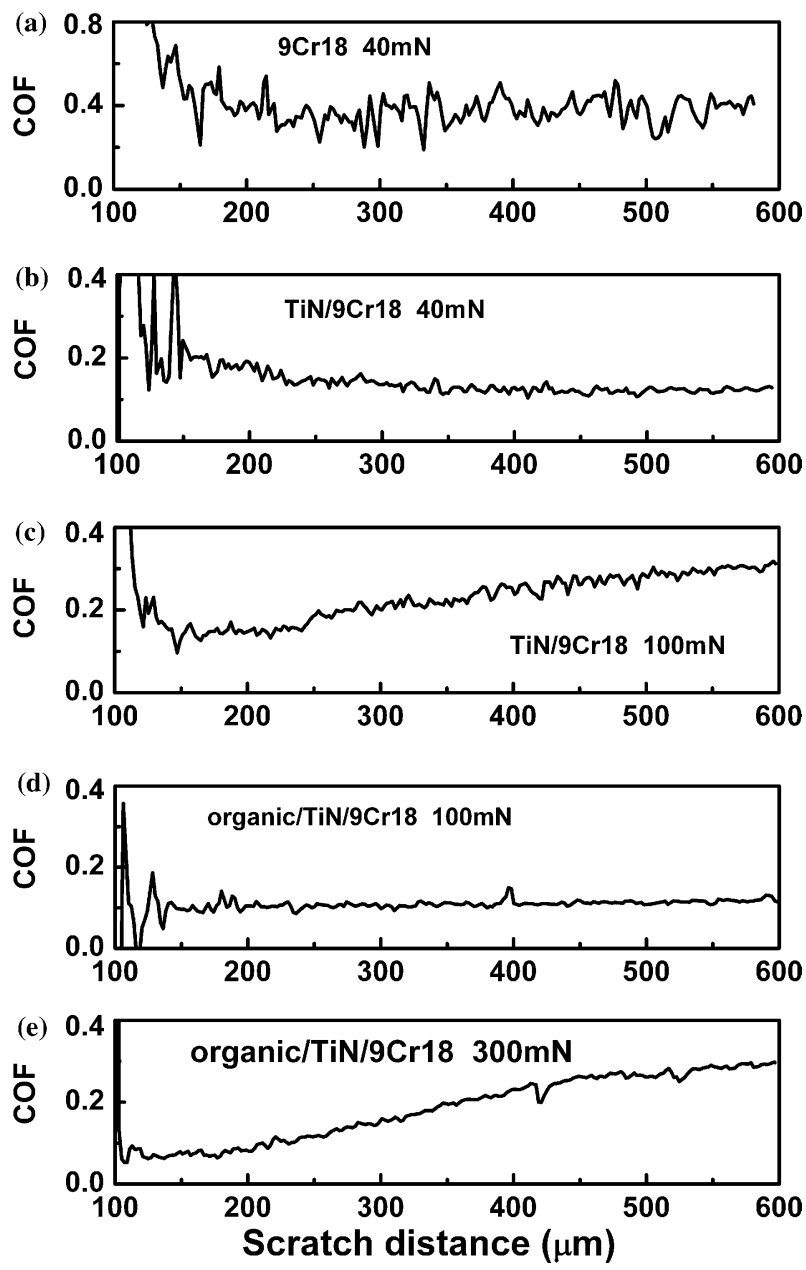

Figure 3. Coefficient of friction as a function of the scratch distance under various ramping normal loads: (a) $9 \mathrm{Cr} 18$ with $40 \mathrm{mN}$; (b) TiN/9Cr18 with $40 \mathrm{mN}$; (c) TiN/9Cr18 with $100 \mathrm{mN}$; (d) organic/

TiN/9Cr18 with $100 \mathrm{mN}$; (e) organic/TiN/9Cr18 with $300 \mathrm{mN}$.

processes occurred successively during the ramping of the normal load up to $300 \mathrm{mN}$ : fully elastic recovery (marked as range L1), plastic deformation (L2), and delamination/fracture (L3) of the films. Figures 4(a)6(a) are the curves for the lateral load as a function of the normal load and scratch distance. The initial point of range L2 corresponds to the commitment of plastic deformation and the initial residual scratching in figures 4(b)-6(b). The normal and lateral loads associated with this event are called, $N_{\mathrm{p}}, F_{\mathrm{p}}$. In addition, the cracking or delamination of a hard coating is marked by a sudden increase in lateral load versus normal load and scratch distance curves. The normal and lateral loads associated with this event are called the critical load, $N_{\mathrm{c}}, F_{\mathrm{c}}$ [11], corresponding to the initial points of the range L3. The data of three films are shown in table 2. The range L1 of organic/TiN/GT35 is the widest, and the values of $N_{\mathrm{p}}$ and $F_{\mathrm{p}}$ are the highest, followed by organic/TiN/9Cr18, and organic/TiN/ 40CrNiMo, showing that TiN/GT35 exhibited the best
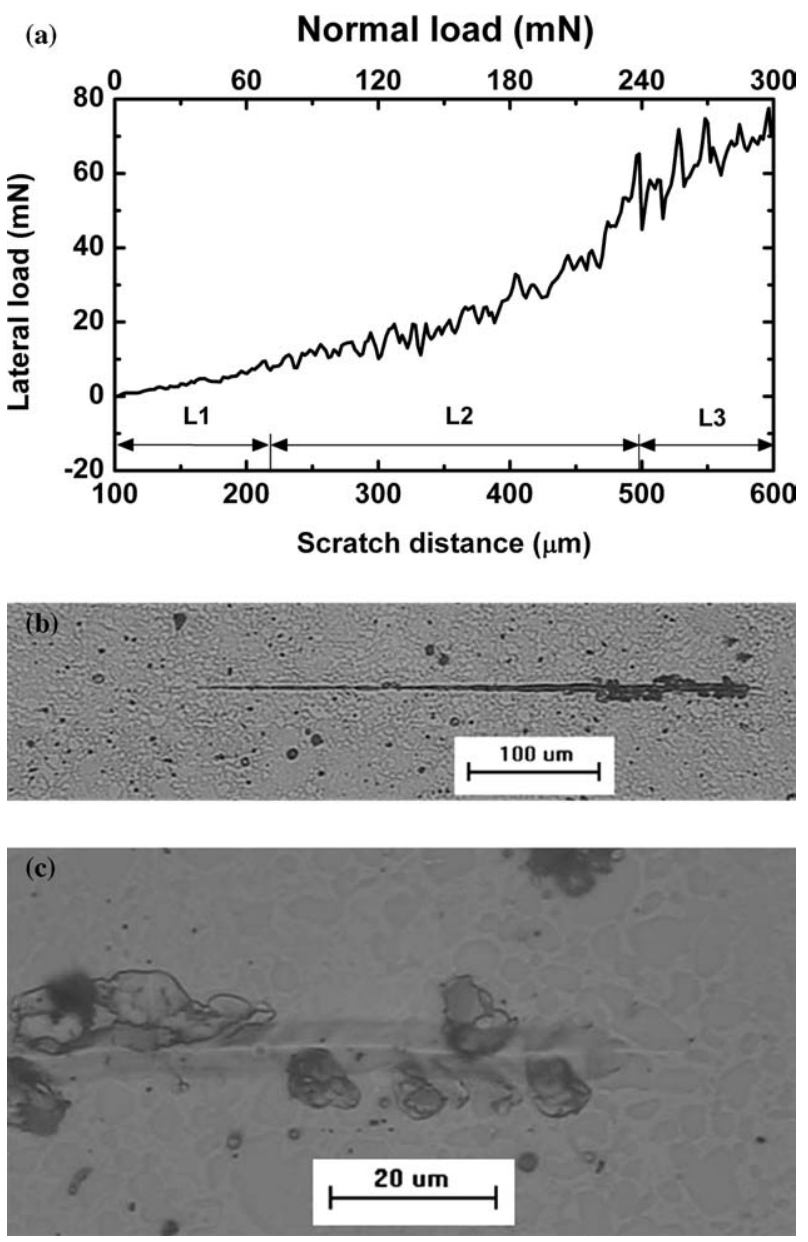

Figure 4. The scratch of organic/TiN/GT35 with ramping normal load of $\max 300 \mathrm{mN}$ : (a) The lateral load as a function of the scratch distance or the normal load; (b) The optical image of the scratch trace; (c) The optical image of the top of the scratch trace.

load-carrying capacity. The range L3 of organic/TiN/ GT35 is the narrowest, and the critical load is the highest, followed by organic/TiN/40CrNiMo, and organic/ TiN/9Cr18, showing that TiN/GT35 also exhibited the best adhesive capacity and scratch/wear resistance.

The damage or failure modes were different for the above samples. It is observed in figures 4(c)-6(c) that the small debris for organic/TiN/GT35 peeled off around the top of the scratch trace, but the small debris of TiN for organic/TiN/9Cr18 and organic/ TiN/40CrNiMo was pressed entirely into the substrates. In particular, the micrograph in figure 6(c) clearly shows that the organic/TiN films were ploughed by the scratch tip, whose scratches were like a fish vertebrae, associated with the plastic flow of $40 \mathrm{CrNiMo}$. For a hard substrate, the main failure mode of TiN film is brittle fracture. The debris of the broken film is larger than that on soft substrates. 
Table 2 .

The deformation range of each sample and the corresponding load.

\begin{tabular}{lccccccc}
\hline & $\mathrm{L}_{1} / \mu \mathrm{m}$ & $\mathrm{L}_{2} / \mu \mathrm{m}$ & $\mathrm{L}_{3} / \mu \mathrm{m}$ & $N_{\mathrm{p}} / \mathrm{mN}$ & $F_{\mathrm{p}} / \mathrm{mN}$ & $N_{\mathrm{c}} / \mathrm{mN}$ & $F_{\mathrm{c}} / \mathrm{mN}$ \\
\hline Organic/TiN/GT35 & $100-220$ & $220-496$ & $496-600$ & 72.0 & 8.0 & 237.6 & 64.8 \\
Organic/TiN/9Cr18 & $100-193$ & $193-417$ & $417-600$ & 55.8 & 4.9 & 190.2 & 46.2 \\
Organic/TiN/40CrNiMo & $100-156$ & $156-440$ & $440-600$ & 33.6 & 2.8 & 204.0 & 96.8 \\
\hline
\end{tabular}

(a)
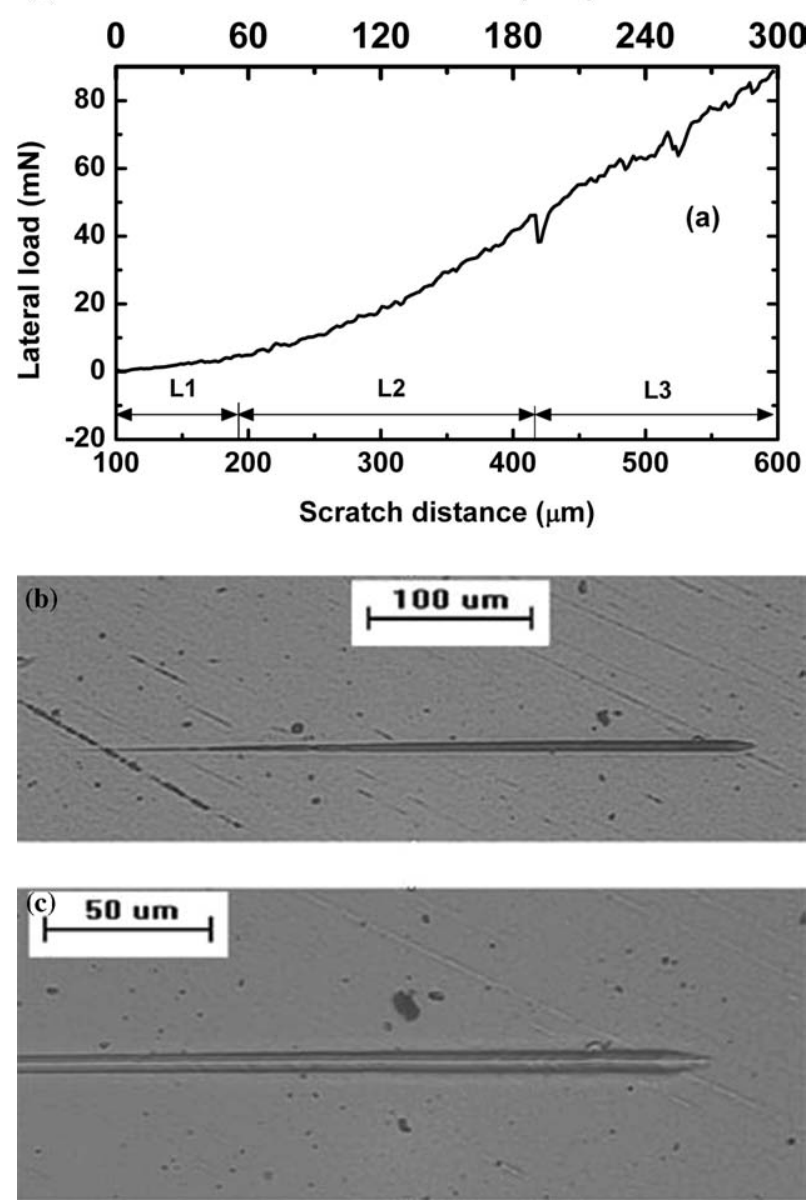

Figure 5. The scratch of organic/TiN/9Cr18 with ramping normal load of $\max 300 \mathrm{mN}$ : (a) The lateral load as a function of the scratch distance or the normal load; (b) The optical image of the scratch trace; (c) The optical image of the top of the scratch trace.

\section{Conclusion}

In this study, we studied the micro/nanomechanical properties of solid-lubricative TiN films on three different types of substrates. The main conclusions may be summarized as follows:

1. Compared with the substrate steels, the mechanical and wear resistance properties of $\mathrm{TiN} /$ steels are significantly improved. The lubricative effects of TiN coatings and the organic coatings are marked.
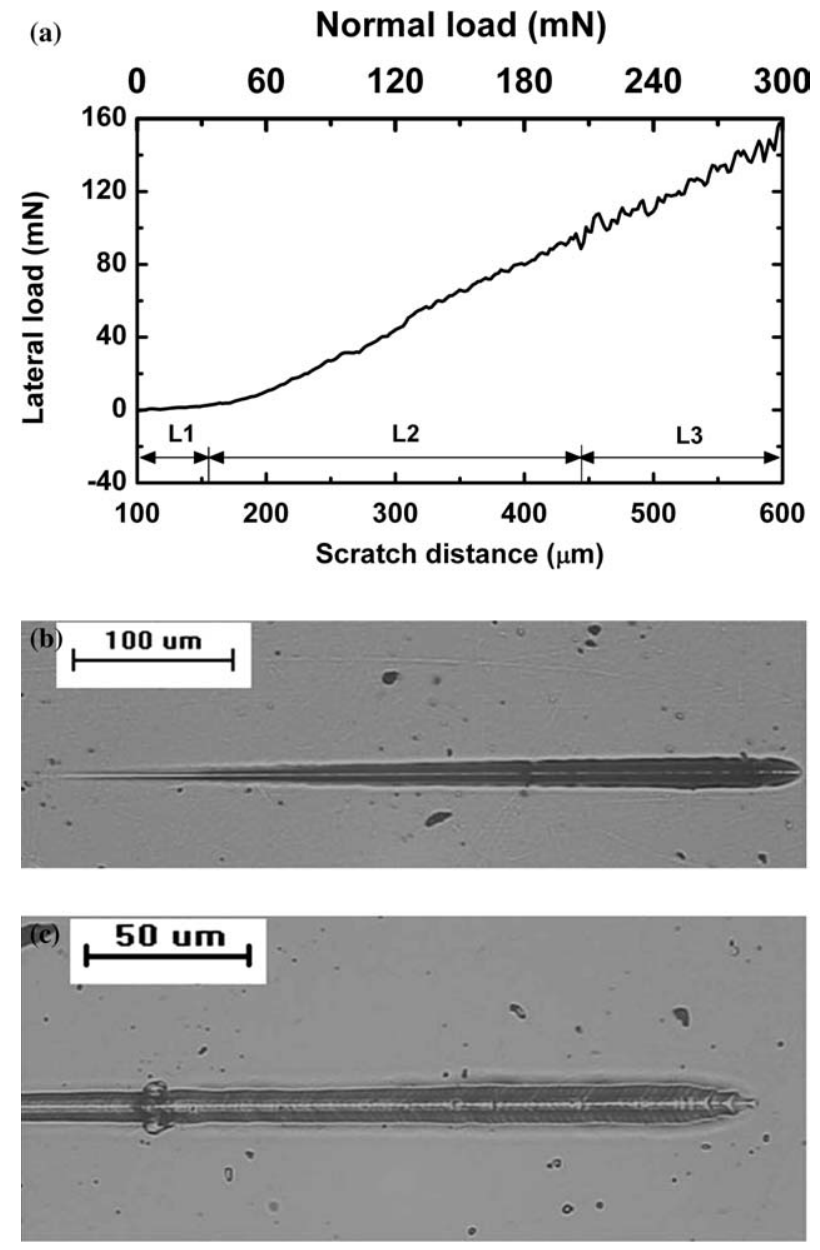

Figure 6. The scratch of organic/TiN/40CrNiMo with ramping normal load of max $300 \mathrm{mN}$ : (a) The lateral load as a function of the scratch distance or normal load; (b) The optical image of the whole scratch trace; (c) The optical image of the top of the scratch trace.

2. GT35 steel is the best substrate. On one hand, the load-carrying capacities of GT35 and TiN/GT35 are the highest. On the other hand, the tendency to fracture of TiN/GT35 is the lowest. This may be due to the interface properties between TiN and GT35 steel, which can reduce the internal stress field and improve the critical load.

3. The nanoindentation and nanoscratch tests can provide very useful information on the near-surface elastic-plastic deformation, fracture, friction and wear properties. 


\section{Acknowledgments}

The authors would like to thank Ms. Xiulan Wang in Beijing Research Institute of Aerospace Materials \& Technology for the preparation of TiN films and Dr. Cunyi Xie in MTS Systems (China) Inc. for helpful discussion. This project was supported by the National Natural Science Foundation of China (10372103 and 10172086) and the Chinese Academy of Sciences (KJCX2-SW-L2).

\section{References}

[1] C. Quaeyhaegens, J. D'Haen, L.M. Stals, et al., Surf. Coat. and Technol. 61 (1993) 227.

[2] K. Xu, J. Chen, R. Gao and J. He, Surf. Coat. and Technol. 58 (1993) 37.
[3] M.G. Hocking, V. Vasantasree, P.S. Sidky, Metallic and Ceramic Coatings: Production, High Temperature Properties and Applications (Wiley, New York, 1989) 86.

[4] J.H. Huang, Y.P. Tsai and G.P. Yu, Thin Solid Films 355/356 (1999) 440.

[5] J.C. Knight, T.F. Page and I.M. Hutchings, Thin Solid Films 177 (1989) 117.

[6] S.V. Hainsworth and W.C. Soh, Surf. Coat. Technol. 163/163 (2003) 515.

[7] W.C. Oliver and G.M. Pharr, J. Mat. Res. 7 (1992) 1564.

[8] C. Charitidis, Y. Panayiotatos and S. Logothetidis, Diam. Rel. Mat. 12 (2003) 1088.

[9] K.W. Lee, Y.-W. Chung, C.Y. Chan, I. Bello, et al., Surf. Coat. Technol. 168 (2003) 57.

[10] N.R. Moody, A. Strojny, D.L. Medlin, et al., J. Mat. Res. 14 (1999) 2306.

[11] B. Bhushan, Handbook of Micro/Nanotribology, 2nd ed. (Boca Raton, CRC Press, 1999). 